\title{
Boosting LINC Systems Combiners Efficiency Through Ring-type Magnitude Modulation
}

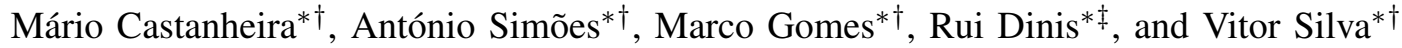 \\ *Instituto de Telecomunicações, Portugal \\ ${ }^{\dagger}$ Department of Electrical and Computer Engineering, University of Coimbra, 3030-290 Coimbra, Portugal \\ $\ddagger$ FCT-UNL, 2829-516 Caparica, Portugal
}

\begin{abstract}
This paper proposes a transmitter structure that combines a ring-type magnitude modulation (RMM) technique with a linear amplification with nonlinear components (LINC) scheme for power and spectrally efficient transmission based on bandwidth limited OQPSK signals, for either a linear combiner (LC) or a Chireix combiner (CC). It shows that by controlling the transmitted signal's envelope through RMM, the range of the LINC decomposition angle is considerably decreased. This significantly improves LC's power efficiency, and substantially reduces CC's spectral leakage while maintaining its high amplification efficiency.
\end{abstract}

Index Terms-LINC, Power Efficiency, Spectral Efficiency, Magnitude Modulation, OQPSK signals, Chireix Combiner, Linear Combiner

\section{INTRODUCTION}

The transmission over mm-wave bands is expected to be a key component of upcoming 5G (Fifth Generation) systems due to the high available band and the small wavelengths that allow massive multiple input, multiple output (MIMO) implementations [1], [2]. However, the transmitter design for these techniques can be particularly challenging, especially for systems requiring high power and spectral efficiencies. In fact, the filtering associated to typical modulations with high spectral efficiency leads to signals with high peak-toaverage power ratio (PAPR) [3], which leads to amplification difficulties.

Strongly nonlinear power amplifiers (such as class D or E amplifiers) are much simpler and more efficient than (quasi)linear amplifiers, but are only suitable for signals with very low envelope fluctuations [4]. By employing linear amplification with nonlinear components (LINC) techniques one can employ strongly nonlinear amplifiers with signals with strong envelope fluctuations [5], [6]. The LINC method consists of separating an input signal in two constant-envelope branches to be amplified separately by two highly efficient strongly non-linear amplifiers, and where the amplified components are subsequently combined in order to produce an output signal corresponding to the linear amplification of the input. However, the wide-range of the LINC phase decomposition angle, $\theta$, for signals with high envelope fluctuations leads to

This work is funded by FCT/MEC through national funds and when applicable co-funded by FEDER - PT2020 partnership agreement under the project UID/EEA/50008/2013. shy power efficiency gains with very good linearity when employing a passive linear combiner (LC), or excellent efficiency at the considerable cost of decreasing linearity when a Chireix outphasing combiner (CC) is used instead [6].

The LINC effectiveness is considerably improved when the signals to be transmitted have smaller envelope fluctuations. As so, the use of offset signals such as OQPSK particularly suits LINC, since it is known that these can have much lower envelope fluctuations than non-offset signals. In fact, recently, new architectures have been proposed for 5G [7], [8] employing multi-layer massive antenna structures allowing for highly power and spectral efficient transmitters leverage on the the use of large constellations (e.g., 16-QAM or 64-QAM) that are decomposed as a sum of several OQPSK-type signals [9]; these OQPSK components can individually be efficiently amplified by using LINC and transmitted separately through different antennas. However, compact spectrum OQPSK signals (e.g., trough a square-root raised-cosine filtering with small roll-off) can possess significant envelope fluctuations which may impact on LINC combiner efficiency; in fact, OQPSK-type signals can only have constant envelope at the expense of bandwidths much wider than the minimum Nyquist band (e.g. GMSK signals) [10]. These facts make it interesting optimising LINC amplification of OQPSK compact spectrum signals.

In this paper we consider the use of LINC transmitters for an efficient power amplification of OQPSK signals with Nyquist filtering. To further reduce the dynamic range of their envelope we employ a ring-type magnitude modulation (RMM) method recently proposed [11]-[13], which decreases considerably the range of the LINC decomposition angle, concentrating $\theta$ 's probability density function (PDF) and shifting it toward lower values, greatly improving the performance of LINC transmitters, reducing the spectral leakage when a $\mathrm{CC}$ is employed, or improving the power efficiency when a LC is used instead. RMM also improves the robustness against gain and phase imbalances between the LINC branches' power amplifiers, and decreases the oversampling requirements of a LINC digital implementation [6], [13].

This paper is organized as follows. Section II presents the architecture of a RMM-LINC system targeted to spectral/power efficient transmission of compact spectrum OQPSK signals. It also presents how the efficiency of LINC is affected by the 


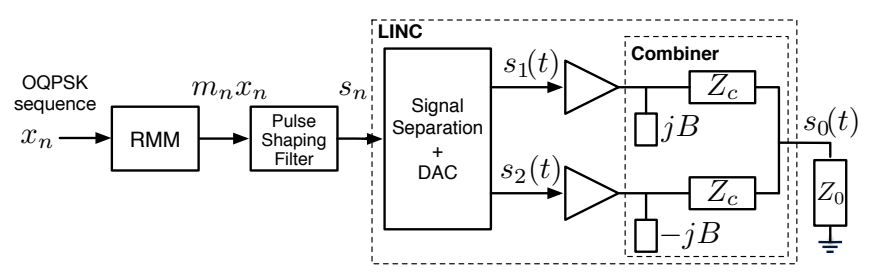

Fig. 1: RMM-LINC transmitter targeted to bandlimited OQPSK transmission.
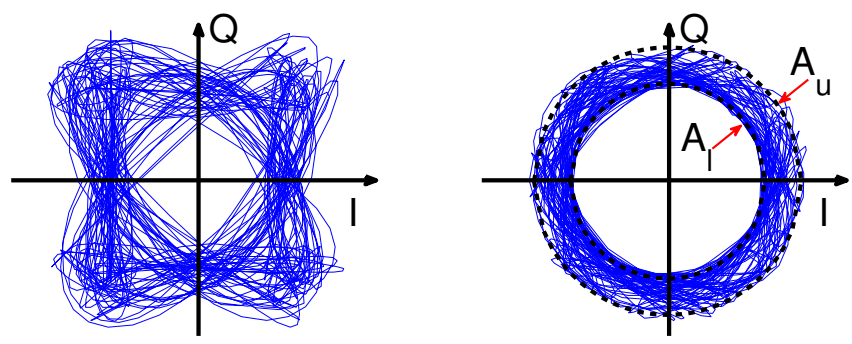

Fig. 2: IQ diagram of $s_{n}$ : (left) without RMM; (right) with RMM.

LINC decomposition angle. Section III addresses LINC optimization through RMM applied to OQPSK signals, especially for LINC using a non-linear Chireix combiner. Results are presented in Section IV and main conclusions summarised in Section V.

\section{THE OQPSK RMM-LINC TRANSMITTER}

Fig. 1 depicts the RMM-LINC transmitter targeted to a spectral/power efficient OQPSK transmission [11], [13]. The bandlimited RMM OQPSK signal $s_{n}$ is given by

$$
s_{n}=\sum_{k}\left(m_{k}^{(I)} x_{k}^{(I)} \delta_{n-k L}+j m_{k}^{(Q)} x_{k}^{(Q)} \delta_{n-k L-\frac{L}{2}}\right) * h_{n},
$$

where $h_{n}$ is the impulse response of the bandwidth-limiting pulse shaping filter designed with oversampling $L$, and $m_{n}^{(I)}$ and $m_{n}^{(Q)}$ are, respectively, the sequences of in-phase and quadrature RMM coefficients applied to the OQPSK symbol sequence, $x_{n}=x_{n}^{(I)}+j x_{n}^{(Q)}$, which guarantee the ring-shape of the amplitude of $s_{n}$, i.e.,

$$
A_{l} \leq\left|s_{n}\right| \leq A_{u}
$$

as shown in Fig. 2, with $A_{l}$ and $A_{u}$ being the lower and upper amplitude boundaries; the RMM coefficients are computed a priori, for the given $h_{n}, A_{l}$, and $A_{u}$, and stored in a LUT [13]. RMM guarantees both a PAPR and envelope dynamic range reduction of the transmitted signal, desirable characteristics for an efficient LINC amplification [6]. Besides, since RMM precedes pulse shaping, efficient spectral confinement is also assured.

The LINC amplification follows [5], [6], which encompasses: $s_{n}$ separation in constant amplitude components $s_{n}^{(1)}$ and $s_{n}^{(2)}$, and their digital-to-analog conversion (DAC) in $s_{1}(t)$ and $s_{2}(t)$; components' high-efficient nonlinear power amplification (NLPA); and signal combining. The signal $s_{n}$ can be generally described in polar form as

$$
s_{n}=r_{n} e^{j \phi_{n}}=s_{n}^{(1)}+s_{n}^{(2)},
$$

with $r_{n}=\left|s_{n}\right|$ and $\phi_{n}=\angle s_{n}$. Thus, with $r_{\max }=\max _{n}\left(r_{n}\right)$, components $s_{n}^{(1)}$ and $s_{n}^{(2)}$ can be written as

$$
s_{n}^{(i)}=\frac{r_{\max }}{2} e^{j\left(\phi_{n}+(-1)^{i} \theta_{n}\right)} \quad \text { for } \quad i \in\{1,2\},
$$

where,

$$
\theta_{n}=\arccos \left(\frac{r_{n}}{r_{\max }}\right)
$$

is the LINC decomposition angle. In order to obtain a linear amplified replica of the input signal $s_{n}=s_{n}^{(1)}+s_{n}^{(2)}$, perfectly balanced amplifiers and a passive LC are needed to amplify and recombine the LINC signal components $s_{1}(t)$ and $s_{2}(t)$.

Despite using highly efficient NLPA, the LINC combining operation's average efficiency, $\bar{\eta}_{c o m b}$, is critical for the transmitter's overall efficiency. For a passive LC, the instantaneous $\eta_{\text {comb }}$ depends exclusively on the decomposition angle $\theta$ [6], as follows:

$$
\eta_{c o m b, L C}=\cos ^{2}(\theta), \quad \theta \in[0 ; \pi / 2],
$$

whereby knowing the $\theta$ 's PDF, i.e. $p(\theta)$, it can easily be computed

$$
\bar{\eta}_{c o m b, L C}=\int_{0}^{\pi / 2} \cos ^{2}(\theta) p(\theta) \mathrm{d} \theta,
$$

and thus, it easy to conclude that efficiency can be improve by reducing the dynamic range of $\theta$ toward lower values.

\section{Chireix OUtPhASING COMBINER}

When amplification linearity is not strictly in demand and power efficiency is of utmost importance, the passive combiner can be replaced by the CC [6], [14] illustrated in Fig. 1; $Z_{c}$ represents the characteristic impedance of the combiner's quarterwave transmission lines, $B$ is the stubs' susceptance, and $Z_{o}$ is the output load's impedance. Leveraging on the RMM method it is possible to considerably mitigate the nonlinear effects associated with $\mathrm{CC}$ while maintaining its excellent power efficiency achieved when it is properly designed.

The CC's instantaneous efficiency, $\eta_{c o m b, C C}$, and the envelope of transmitted signal $s_{o}(t)$ can be evaluated as a function of the circuit's electrical parameters [15], namely, the stubs' electrical length $\gamma=\arctan \left(B \cdot Z_{0}\right)$ and the normalized characteristic impedance $z_{c}=Z_{c} / Z_{o}$, by using the experimentally validated Chireix model described in [14]; these electrical parameters account for the unmatched and nonisolated nature of the $\mathrm{CC}$, and the reflection phenomena associated with it. Thus, it comes that

$$
\eta_{c o m b, C C}\left(\theta, \gamma, z_{c}\right)=\frac{8 z_{c}^{2} \cos ^{2}(\gamma)}{\left(z_{c}^{2}+2 \cos ^{2}(\gamma)\right)^{2}} \cos ^{2}(\theta-\gamma) .
$$


As in the passive LC case, the LINC's decomposition angle $\theta$ affects $\eta_{c o m b, C C}$, which can be rewritten as $\eta_{c o m b, C C}=K \cos ^{2}(\theta-\gamma)$, where

$$
K\left(\gamma, z_{c}\right)=\frac{8 z_{c}^{2} \cos ^{2}(\gamma)}{\left(z_{c}^{2}+2 \cos ^{2}(\gamma)\right)^{2}}
$$

is a constant term that depends only on the circuit's electrical parameters, and that can be maximized, i.e., $K\left(\gamma, z_{c}\right)=1$ [14], which is attained for

$$
z_{c}=\sqrt{2} \cos (\gamma) .
$$

Thus, the maximization of the average Chireix's power efficiency, $\bar{\eta}_{c o m b, C C}$ depends only on the proper design of stubs' electrical length $\gamma$ targeted to a given $\theta$ 's distribution. In fact, since $K$ can always be set to 1 for some $\gamma$ through (10), by focusing on the term $\cos ^{2}(\theta-\gamma)$ in (8), $\gamma$ should be selected in order to shift $\eta_{c o m b, C C}$ curve towards the more likely values of $\theta$. In [14] it is proposed to use the PDF of the modulated signal in order to identify the average signal level, and the correspondent LINC decomposition angle, $\bar{\theta}$, and then setting $\gamma=\bar{\theta}$ and $z_{c}$ accordingly. However, the PDF of $\theta$ can be used instead to maximize $\bar{\eta}_{c o m b, C C}$ following

$$
\max \left\{\bar{\eta}_{c o m b, C C}\right\}=\max _{\gamma} \int_{0}^{\pi / 2} \cos ^{2}(\theta-\gamma) p(\theta) \mathrm{d} \theta .
$$

The stubs' length, $\gamma_{o p t}$, can be found by differentiating (11) with respect to $\gamma$ and find the resulting expression's zero value:

$$
\begin{array}{r}
\frac{d}{d_{\gamma}} \int_{0}^{\frac{\pi}{2}} \cos ^{2}(\theta-\gamma) p_{\theta}(\theta) d \theta=0 \Leftrightarrow \\
\int_{0}^{\frac{\pi}{2}} \sin (2(\theta-\gamma)) p_{\theta}(\theta) d \theta=0 .
\end{array}
$$

Analysis of (8) and (12) shows that the reduction of the dynamic range of $\theta$ allows an increase on the achievable instantaneous and maximum average efficiency. By reducing the envelope fluctuations and guaranteeing $A_{l} \leq\left|s_{n}\right| \leq A_{u}$, it considerable reduces the range of $\theta$ possible values, wherefore minimizing the difference $\theta-\gamma_{o p t}$ and making $\cos ^{2}\left(\theta-\gamma_{o p t}\right)$ approach to 1 . This advantage is also significant for the passive LC, with efficiency given by (6) and (7), where the $\cos ^{2}(\theta)$ behaviour makes avoiding large values of $\theta$ very important.

However, regarding $\mathrm{CC}$, the major advantages on the use of RMM relates to a considerable increase of combiners' linearity. While the use of the susceptance $B=\tan \left(\gamma_{o p t}\right) / Z_{o}$ in each of the LINC's branches improves the combiner's efficiency, this improvement is obtained at the expense of amplification linearity. Accordingly to [14], the envelope $r_{o}$ of the recombined signal $s_{o}(t)$ can be written as

$$
r_{o, C C}=\frac{2 z_{c} G \cos (\gamma)}{z_{c}^{2}+2 \cos ^{2}(\gamma)} r_{\max } \cos (\theta-\gamma),
$$

where $G$ is the LINC branches amplifiers' gain. Note that according to the LINC decomposition described by (4), for a passive linear combiner

$$
r_{o, L C}=G r_{\max } \cos (\theta) .
$$

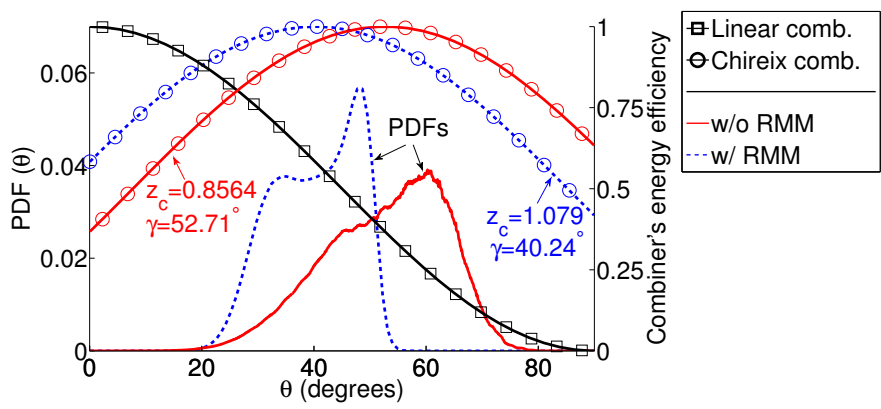

Fig. 3: $\theta$ 's PDF and $\eta_{\text {comb }}$ (optimized) for the OQPSK RMM-LINC Transmitter.

TABLE I: Average power efficiency, $\bar{\eta}_{\text {comb }}$, for different LINC combiners.

\begin{tabular}{|l|cc|}
\hline$\eta_{\text {comb }}$ & w/o RMM & RMM \\
\hline Linear & $37.6 \%$ & $58.0 \%$ \\
\hline Chireix & $96.6 \%$ & $98.3 \%$ \\
\hline
\end{tabular}

Thus, while the first term of (13) is constant for a given electrical circuit, the second term is a shifted version of (14), which means that there is a nonlinear amplification gain that results from using the CC. Here is where RMM plays a key role; even though the nonlinear effects introduced by the CC cannot be completely avoided, they can be significantly mitigated by reducing the range of the decomposition angle $\theta$, which is achieved though RMM.

\section{NUMERICAL RESULTS}

The effect of combining the RMM technique in a LINC transmitter scheme that employs either a CC or LC is presented. We study the proposed transmitter's average power efficiency, bit error rate (BER) over an AWGN channel, and power spectral density (PSD) of the transmitted signals. We employed a pulse shaping root-raised cosine filter with a rolloff factor of $25 \%$, a $(1680,840)$ LDPC code for channel error control coding, and an RMM block with amplitude boundaries $A_{l}=0.8$ and $A_{u}=1.1$ (for a normalized OQPSK constellation, i.e., $\left|x_{n}\right|=1$ ). Fig. 3 shows the related $\theta$ 's PDF for a transmission with and without RMM. By following the procedure described above, it was determined that for the conventional OQPSK LINC transmitter the optimal Chireix circuit's electrical parameters are $z_{c}=0.8564$ and $\gamma=52.71^{\circ}$, and become $z_{c}=1.079$ and $\gamma=40.24^{\circ}$ when RMM is employed, with the correspondent $\eta_{c o m b}$ curves being shown, also, in Fig. 3. Note that, as expected, the maximum of $\eta_{c o m b, C C}$ 's function shifts toward the most probable values of $\theta$. It is also visible the reduction on $\theta$ 's range produced by RMM and the avoidance of high $\theta$ 's values; from (7) and (11), it results, as shown in Table I, in an expected improvement of $\bar{\eta}_{\text {comb }}$, specially in the LC scenario.

The major advantages of RMM appear when analysing the PSD of the transmitted signal and the BER as function of $E_{b} / N_{0}$ (energy per information bit to noise power spectral density ratio) compensated by the combiner's power losses, as shown in Figs. 4 and 5. For the LC there is $0.55 \mathrm{~dB}$ 


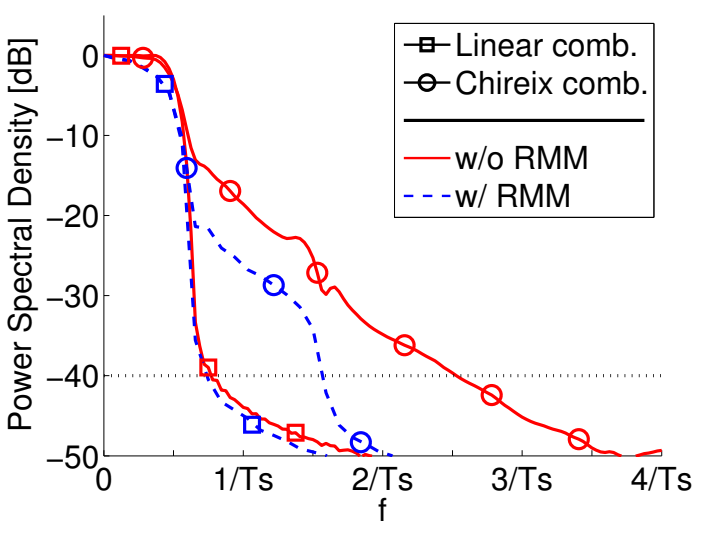

Fig. 4: PSD for different the OQPSK LINC Transmitters $\left(T_{s}\right.$ denotes the symbol period).

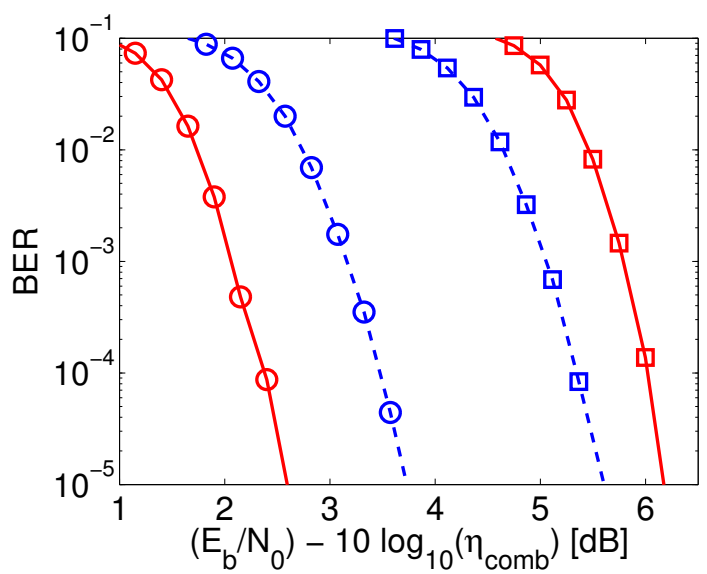

Fig. 5: BER for different the OQPSK LINC Transmitters. (Note: For better clarity, legend is omitted and it is considered to be same as used in Fig. 4.)

BER's gain while keeping spectral confinement (remember that RMM precedes pulse-shapping). Also observable is the major power efficiency advantage of using the $\mathrm{CC}$ at the cost of a considerable spectral leakage. The use of RMM plays here a key compromise's role. Although the increase on $\bar{\eta}_{c o m b, C C}$ is not sufficient to overcompensate the symbol's added distortion due to RMM procedure [11], [13] (as verified for the LC where there is a huge gain on combiner efficiency) resulting in a loss of $1 \mathrm{~dB}$ at BER performance compared to typical transmitter employing the $\mathrm{CC}$ (see Fig. 5), the spectral leakage is notably reduced by $50 \%$, taking as reference the bandwidth at $40 \mathrm{~dB}$ of attenuation (see Fig. 4); note that, the BER performance here is still much higher than for the LC with RMM, enhancing the considerable bandwidth saving obtained through RMM for LINC with a CC.

\section{CONCLUSiON}

This paper shows that RMM can effectively enhance the efficiency of bandwidth limited OQPSK transmissions using LINC amplification. Considering LINC employing a CC, it shows that RMM can produce considerable bandwidth savings (reducing spectral leakage about 50\%) while still granting a very high power efficiency, by reducing the nonlinear effects experienced by the transmitted signal's envelope that are introduced by the CC. Also, for the LC case, the combiner power efficiency is significantly improved with high BER gains.

\section{REFERENCES}

[1] T. S. Rappaport, S. Sun, R. Mayzus, H. Zhao, Y. Azar, K. Wang, G. N. Wong, J. K. Schulz, M. Samimi, and F. Gutierrez, "Millimeter Wave Mobile Communications for 5G Cellular: It Will Work!" IEEE Access, vol. 1, pp. 335-349, 2013.

[2] S. Rangan, T. Rappaport, and E. Erkip, "Millimeter-Wave Cellular Wireless Networks: Potentials and Challenges," Proceedings of the IEEE, vol. 102, no. 3, pp. 366-385, March 2014.

[3] G. Wunder, R. F. H. Fischer, H. Boche, S. Litsyn, and J. S. No, "The PAPR Problem in OFDM Transmission: New Directions for a LongLasting Problem," IEEE Signal Processing Magazine, vol. 30, no. 6, pp. 130-144, Nov 2013.

[4] P. Reynaert and M. Steyaert, RF Power Amplifiers for Mobile Communications. Springer, 2006

[5] D. Cox, "Linear Amplification with Nonlinear Components," Соттиnications, IEEE Transactions on, vol. 22, no. 12, pp. 1942-1945, Dec 1974.

[6] A. Birafane, M. El-Asmar, A. Kouki, M. Helaoui, and F. Ghannouchi, "Analyzing LINC systems," Microwave Magazine, IEEE, vol. 11, no. 5, pp. 59-71, Aug 2010.

[7] R. Dinis, P. Montezuma, P. Bento, M. Gomes, and V. Silva, "A multiantenna technique for mm-wave communications with large constellations and strongly nonlinear amplifiers," in Microwave Conference (GeMiC), 2015 German, March 2015, pp. 284-287.

[8] _ - "A Massive MIMO Architecture for Highly Efficient mm-Wave Communications with Saturated Amplifiers," in International Conference on Electronics, Information, and Communication (ICEIC 2015), January 2015.

[9] R. Dinis, P. Montezuma, N. Souto, and J. Silva, "Iterative FrequencyDomain Equalization for general constellations," in Sarnoff Symposium, 2010 IEEE, April 2010, pp. 1-5.

[10] A. Carlson and P. Crilly, Communication Systems. McGraw-Hill, 2009.

[11] M. Gomes, V. Silva, F. Cercas, and M. Tomlinson, "Power efficient backoff reduction through polyphase filtering magnitude modulation," IEEE Communications Letters, vol. 13, no. 8, pp. 606-608, August 2009.

[12] A. Simões, M. Gomes, R. Dinis, V. Silva, and F. Cercas, "Magnitude Modulation applied to LINC transmitters: Paving the Road for Better Efficiency," IEEE 80th Vehicular Technology Conference: VTC2014Fall, Sep 2014.

[13] A. Simoes, P. Bento, M. Gomes, R. Dinis, and V. Silva, "Efficient LINC Amplification for 5G through Ring-Type Magnitude Modulation," in 2015 IEEE Globecom Workshops (GC Wkshps), Dec 2015, pp. 1-6.

[14] M. El-Asmar, A. Birafane, M. Helaoui, A. Kouki, and F. Ghannouchi, "Analytical design methodology of outphasing amplification systems using a new simplified chireix combiner model," Microwave Theory and Techniques, IEEE Transactions on, vol. 60, no. 6, pp. 1886-1895, June 2012.

[15] A. Birafane and A. B. Kouki, "On the linearity and efficiency of outphasing microwave amplifiers," IEEE Transactions on Microwave Theory and Techniques, vol. 52, no. 7, pp. 1702-1708, July 2004. 